\title{
Tomada de decisão em dependentes de crack: um estudo com o Iowa Gambling Task
}

\author{
Thiago Wendt Viola \\ Caroline de Oliveira Cardoso \\ Ingrid D'Avila Francke \\ Hosana Alves Gonçalves \\ Pontifícia Universidade Católica do Rio Grande do Sul \\ Julio Carlos Pezzi \\ Universidade Federal de Ciências da Saúde de Porto Alegre \\ Renata Brasil Araújo \\ Hospital Psiquiátrico São Pedro \\ Rochele Paz Fonseca \\ Rodrigo Grassi-Oliveira \\ Pontifícia Universidade Católica do Rio Grande do Sul
}

\begin{abstract}
Resumo
Este estudo investigou como ocorre o processo de tomada de decisão em dependentes de crack pelo instrumento Iowa Gambling Task (IGT). Foram selecionados 30 participantes para o grupo de dependentes de crack - GDC, e 15 controles não usuários - GNU, de ambos os sexos. Para avaliar a intensidade de craving utilizou-se o Cocaine Craving Questionnaire-Brief. Houve diferenças significativas entre os grupos tanto no cálculo total, como no cálculo por blocos. A curva de aprendizagem do GDC manteve-se constante e negativa na maior parte do jogo, havendo apenas no final um indício de aprendizagem. Em relação à classificação do desempenho na tarefa, as análises evidenciaram que um significativo número de participantes controles obtiveram desempenho não-prejudicado, oposto ao desempenho do GDC. As diferenças entre os grupos investigadas no IGT corroboraram com achado de estudo anterior, que evidenciou prejuízo no processo de tomada de decisão associado à dependência de cocaína e de crack.
\end{abstract}

Palavras-chave: crack; cocaína; testes neuropsicológicos; tomada de decisão; processos cognitivos.

\begin{abstract}
Decision making in addiction to crack: a study with the Iowa Gambling Task. This study investigated how decision-making process occurs in crack dependents through the Iowa Gambling Task (IGT). 30 participants were selected to crack dependent group - GDC, and 15 non-users controls - GNU, from both sexes. We used the Cocaine Craving Questionnaire-Brief to assess the craving intensity. There were significant differences between groups both in the total-calculus score and in the blocks scores. The learning curve of the GDC was constant and negative during almost all game, except in the very ending when a suggestion of learning was observed. Regarding the task performance's classification, the analysis showed that a significant number of controls participants achieved a non-impaired performance, opposed to GDC performance. The differences between groups investigated in the IGT corroborate with a previous study finding, about a worse decisionmaking process associated with cocaine and crack addiction.
\end{abstract}

Keywords: crack; cocaine; neuropsychological tests; decision making; cognitive processes.

$\mathrm{C}$ om a progressiva disseminação do crack, um subproduto da cocaína em forma de pedra, iniciou-se, segundo especialistas, uma epidemia séria com muitas consequências para a saúde pública (Duailibi, Ribeiro, \&
Laranjeira, 2008), resultando em um aumento das demandas dos serviços e unidades especializadas em dependência de substâncias (Nappo, Galduroz, Raymundo, \& Carlini, 2001). Considerando a perspectiva neurocognitiva associada ao 
consumo crônico de cocaína, diversos autores sugerem que o uso abusivo desta substância e de seus derivados pode impactar negativamente o desempenho de diversas funções cognitivas (Berry et al., 1993; Rosselli, Ardila, Lubomski, Murray, \& King, 2001; Severtson, Hedden, Martins, \& Latimer, 2010).

Por meio de estudos neuropsicológicos, recentemente foi evidenciado que a dependência de cocaína está associada a déficits cognitivos em funções específicas do funcionamento do indivíduo, sobretudo aquelas que permitem a adaptação ao ambiente e às suas distintas variações (Cunha, Nicastri, Gomes, Moino, \& Peluso, 2004). Essas funções são denominadas funções executivas (FE) e são conhecidas por desempenharem atividades complexas e orientadas a um objetivo. Ainda, sabese que as FE estão particularmente associadas ao lobo frontal e às suas conexões (Funahashi, 2001; Tirapu-Ustárroz, GarcíaMolina, Luna-Lario, Roig-Rovira, \& Pelegrín-Valero, 2008). Em geral, disfunções executivas impactam na coordenação das funções cognitivas básicas, e são usualmente caracterizadas por prejuízos de iniciação, planejamento, sequenciamento, inibição, flexibilidade, e/ou monitoramento (Levine et al., 2011). No entanto, também estão relacionadas à habilidade de resolver problemas e, sobretudo, à capacidade de tomada de decisão do indivíduo (Toplak, Sorge, Benoit, West, \& Stanovich, 2010). Para alguns autores, a tomada de decisão pode ser considerada até mesmo um componente específico das FE (Chan, Shum, Toulopoulou, \& Chen, 2008).

A tomada de decisão, sob a visão cognitiva, é definida como uma função complexa que envolve a escolha de uma ou mais opções dentre as alternativas vigentes, valorizando sua influência em futuras ações (Naqvi \& Bechara, 2006; Tversky \& Kahneman, 1974). Bechara (1997), em seus estudos com pacientes com lesão frontal, amplia esse conceito ao considerar a influência da emoção e de seus substratos neurológicos nesses processos. Deste modo, a predisposição por opções e reações rápidas, não planejadas, sem considerar as consequências negativas de tais escolhas foi inicialmente evidenciada em pacientes com lesões cerebrais no córtex pré-frontal ventrolateral (Bechara, Damasio, Damasio, \& Anderson, 1994). A partir de estudos com esses pacientes, Bechara, Tranel e Damasio (2000) desenvolveram o instrumento neuropsicológico Iowa Gambling Task (IGT), com o intuito de avaliar o processo de tomada de decisão sob a incerteza (Bechara, Damasio, Damasio, \& Lee, 1999; Bechara, Damasio, Tranel, \& Damasio, 2005). O IGT examina o comportamento do indivíduo em um jogo de cartas composto por quatro baralhos. Ao longo de 100 jogadas, o examinando deve escolher cartas dentre quatro baralhos, dois mais vantajosos e dois desvantajosos, com o objetivo de acumular o máximo de dinheiro possível (Bechara et al., 1994).

Através da análise da tomada de decisão por meio do IGT, alguns autores propuseram a Hipótese do Marcador Somático (Damasio, 1996), sugerindo que antes de se fazer uma análise de custo e benefício das escolhas, o indivíduo se depara com um estado afetivo, ou melhor, com uma sensação corporal automática, conhecida como um conjunto de "marcadores somáticos" (Bechara et al., 2005; Verdejo-Garcia \& Bechara, 2009). Essas respostas corporais são sinais emocionais que antecipam as possíveis consequências da alternativa optada, e acabam por induzir e agilizar a decisão. Desta maneira, a eleição do que é vantajoso ou desvantajoso é baseada nas experiências passadas de cada indivíduo, naquilo que foi sendo registrado ao longo de sua vida como prazeroso ou desprazeroso (Cardoso et al., 2010).

Além da aplicação em pacientes neurológicos, o IGT vem se mostrando sensível para identificar prejuízos no processo de tomada de decisão de diferentes populações psiquiátricas, tal como em dependentes de substâncias (Bechara \& Damasio, 2002). Nesse sentido, investigações recentes sugerem que a alteração do processo decisional pode implicar um conjunto de consequências de fundamental importância para o curso da dependência de substâncias, como a recaída e uma possível não adesão ao tratamento (Bechara, 2005; Bechara \& Van Der Linden, 2005). Em um estudo realizado por Stout, Busemeyer, Lin, Grant e Bonson (2004), o IGT foi administrado a 12 dependentes de cocaína e 14 não usuários. Os resultados indicaram que os participantes dependentes químicos optaram, repetidas vezes, pelos cartões dos baralhos não vantajosos, ao contrário dos participantes controles. Os autores concluíram que os usuários de cocaína apresentaram um déficit significativo na tomada de decisão. Do mesmo modo, Verdejo-García e colaboradores (2007) compararam o desempenho de dependentes de cocaína $(n=12)$, com usuários abusivos de maconha $(n=$ 11) e participantes não usuários de substâncias psicoativas (n =14). Igualmente, os usuários de cocaína selecionaram uma maior proporção de cartas dos baralhos desvantajosos, em relação aos usuários de maconha e aos participantes controles. De forma geral, os dependentes de cocaína demonstram, no IGT, um padrão de sobrepostas escolhas desvantajosas em diversas situações, habitualmente priorizando ganhos em curto prazo, com tendências impulsivas, sem premeditação ou julgamento (Kjome et al., 2010), e isso estaria congruente com o funcionamento do indivíduo dependente, que mantém o comportamento de uso da droga, a despeito de suas consequências negativas, seja na área social, psicológica ou legal (APA, 2002).

Surpreendentemente, mesmo em face das possíveis alterações de tomada de decisão na dependência de crack e do fato de o IGT ser um dos padrões-ouro para exame deste processamento, apenas um estudo se propôs a investigar o processo de tomada de decisão pelo IGT em uma população de usuários de crack $(n=15)$, comparando-os a um grupo composto por não usuários $(n=15)$ (Cunha, Bechara, de Andrade, \& Nicastri, 2011). Os autores concluíram que os dependentes de crack apresentaram prejuízos no processo de tomada de decisão. Além de optarem por escolhas mais desadaptativas no IGT, a evolução da curva de aprendizagem do grupo de dependentes foi diferente quando comparada ao grupo controle: os dependentes demonstraram uma incapacidade de apreender com seus erros, refletindo em uma "miopia para o futuro".

Frente à evidente escassez de estudos que busquem caracterizar o processamento de tomada de decisão associado à dependência de crack, até mesmo em nível internacional, considerando do mesmo modo a utilização do paradigma do IGT e à participação de amostras maiores, o objetivo do presente estudo foi investigar se há déficits na tomada de decisão por meio de tal instrumento, em uma amostra composta por dependentes 
de crack, comparando o desempenho destes a um grupo controle composto por não usuários.

\section{Método}

\section{Delineamento}

Esse é um estudo transversal comparativo entre um grupo de usuários de crack e um grupo controle de não usuários em uma tarefa que avalia a tomada de decisão.

\section{Participantes}

Quarenta e cinco adultos participaram deste estudo. Foram selecionados 30 participantes para o grupo de dependentes de crack - GDC, sendo 15 homens e 15 mulheres, que atenderam aos critérios diagnósticos para dependência de cocaína tipo crack segundo o Diagnostic and Statistical Manual of Mental Disorders - DSM-IV-TR (APA, 2002) no momento de admissão em uma unidade de internação psiquiátrica para desintoxicação. O crack deveria ser a droga de preferência, não sendo excluídos os sujeitos que dependessem de outras substâncias psicoativas, já que é característica da amostra pesquisada o uso de polisubstâncias.

O GDC foi recrutado em duas unidades para desintoxicação de usuários de substâncias, em Porto Alegre (Rio Grande do Sul, Brasil). Ambas as Unidades fazem parte do Sistema Único de Saúde (SUS). Os seguintes critérios de inclusão nortearam a seleção final da amostra: ter idade entre 18 e 59 anos, estar internados há pelo menos duas semanas para desintoxicação de cocaína tipo crack. Os critérios de exclusão foram (a) diagnóstico clínico de retardo mental e/ou demência, ou indicativo de prejuízo cognitivo grave segundo o Mini Exame do Estado Mental (Folstein, Folstein, \& McHugh, 1975; Maia et al., 2006); (b) história atual de outras doenças sistêmicas (incluindo hipertensão, doenças inflamatórias ou infecciosas) ou neurológicas; (c) uso de medicamentos capazes de induzir manifestações psicopatológicas ou alterações cognitivas significantes (por exemplo, benzodiazepínicos).

Para efeito de comparação, um grupo controle de voluntários saudáveis e não usuários - GNU $(n=15$, sendo 8 homens e 7 mulheres) foi recrutado, por conveniência, na cidade de Porto Alegre. A seleção obedeceu aos seguintes critérios de inclusão: (1) idade média semelhante ao grupo clínico, (2) escolaridade média semelhante ao grupo clínico e (3) pertencer à classe econômica nível $\mathrm{C}$ de acordo com a Associação Brasileira de Empresas de Pesquisas (ABEP). Os critérios de exclusão empregados para o GNU foram: (1) atender algum critério de uso, abuso ou dependência de substância; (2) demais critérios de exclusão utilizados para o grupo clínico.

\section{Procedimentos e instrumentos}

$\mathrm{O}$ estudo priorizou seguir as condições estabelecidas na Resolução 196/96 do Conselho Nacional de Saúde (CNS), sendo aprovado pelo Comitê de Ética e Pesquisa da Pontifícia Universidade Católica do Rio Grande do Sul, Hospital Mãe de Deus e Hospital Psiquiátrico São Pedro. Todos os participantes assinaram um termo de consentimento livre e esclarecido (TCLE) previamente ao estudo, estando cientes dos procedimentos da pesquisa e livres para decidirem em qualquer momento sobre sua descontinuidade. Após o consentimento, os participantes foram entrevistados pelos pesquisadores acerca de questões referentes a dados sociodemográficos e uso de substâncias. A entrevista realizada contemplava informações de identificação e socioeconômicas, tempo de internação, bem como questões associadas ao histórico médico. No que se refere ao uso de substâncias, os pesquisadores investigaram cuidadosamente, por meio de uma ficha auxiliar estruturada, a história de uso de drogas, incluindo o período de início, a data do último uso e período de abstinência. Esses dados são apresentados nas Tabelas 1 e 2 . Todos os instrumentos foram administrados em sessão única.

O Cocaine Craving Questionnaire-Brief (CCQ-B) (Iraurgi Castillo, Trujols Albet, Jimenez-Lerma, \& Landabaso, 2009), adaptado ao Brasil (Araujo, Pedroso, \& Castro, 2010), foi utilizado para avaliar se a intensidade do desejo de usar crack seria uma variável interveniente nos resultados do IGT (Cordovil de Sousa Uva et al., 2010). Este instrumento é composto por 10 questões, sendo 8 referentes a intensidade do craving e 2 referentes a resistência ao craving. As respostas são oferecidas em escala do tipo Likert de 0-7 pontos, de discordo totalmente a concordo totalmente. Para o presente estudo utilizou-se a análise das questões referentes à intensidade do craving, por meio da soma das 8 questões correspondentes.

O IGT, como citado anteriormente, é um instrumento utilizado para avaliar a tomada de decisão. Sua versão original foi traduzida, adaptada e validada para o Sul do Brasil (Schneider \& Parente, 2006) e apresenta, junto a isso, evidências de fidedignidade (Cardoso et al., 2010) e de validade para população local (Carvalho, 2010). Os participantes foram instruídos a escolher uma carta por vez, clicando em qualquer baralho que escolher. Visto que o objetivo do jogo é de ganhar o máximo de dinheiro possível, igualmente evitando perder o máximo de dinheiro, uma única dica foi dada aos participantes: dentre os quatro baralhos de cartas, existem alguns que são piores que os outros e, para ganhar, precisa se manter afastado dos baralhos ruins. Dessa forma, ao longo do jogo o participante se depara com dois baralhos vantajosos (C e D), uma vez que resultam em ganhos monetários em longo prazo e baixa perda de dinheiro, e os outros dois baralhos desvantajosos (A e B), que trazem ganhos de muito dinheiro em curto prazo, porém com perda monetária mais frequente e intensa. Duas pontuações mais utilizadas na literatura foram utilizadas. A primeira, "cálculo total", corresponde à soma de todas as escolhas dos baralhos vantajosos $(\mathrm{C}+\mathrm{D})$ menos a soma dos baralhos considerados de risco $(\mathrm{A}+\mathrm{B})$. A classificação do desempenho decisório dos participantes em adaptativo, limítrofe ou prejudicado se dá conforme os pontos de corte adotados por Denburg, Tranel e Bechara (2005). No caso de escores superiores a 18, o desempenho decisional é classificado como não prejudicado; entre -18 e 18, limítrofe; e inferiores a -18, prejudicado. A segunda pontuação indica o quanto houve de aprendizagem ao longo da tarefa e é denominada "cálculo por blocos", permitindo a análise de uma curva de aprendizagem. $\mathrm{O}$ mesmo cálculo é então realizado para cada um dos 5 blocos de 
20 jogadas (Bechara et al., 1994; Bechara et al., 2000).

\section{Análise estatística}

Os dados foram organizados em um banco de dados e analisados através do programa SPSS for Windows 17.0. Os testes estatísticos utilizados estão descritos conforme a característica de cada variável. A normalidade das variáveis foi avaliada para todas as variáveis e quando necessário, os dados foram submetidos à transformação logarítmica.

Para testar a ocorrência de diferenças entre as variáveis

Tabela 1

Caracterização da amostra quanto à idade, escolaridade e gênero

\begin{tabular}{|c|c|c|c|}
\hline \multirow{2}{*}{ Variável } & $\operatorname{GDC}(N=30)$ & GNU $(N=15)$ & \multirow[b]{2}{*}{$p$} \\
\hline & $M, N(D P ; \%)$ & $M, N(D P ; \%)$ & \\
\hline Idade (anos) & $30,37(10,06)$ & $27,20(7,47)$ & n.s. \\
\hline Escolaridade $^{1}$ (anos) & $7,77(2,28)$ & $8,47(2,10)$ & n.s. \\
\hline Homens & $15(65,21)$ & $8(34,78)$ & n.s. \\
\hline Mulheres & $15(68,18)$ & $7(31,81)$ & n.s. \\
\hline
\end{tabular}

Nota: GDC - grupos dependentes de crack; GNU- grupo não usuário

${ }^{1}$ Anos de ensino formal sem repetência

Tabela 2

Padrão de uso de substâncias dos participantes dependentes de crack

\begin{tabular}{lrrr}
\hline & $N$ & \multicolumn{1}{c}{$M$} & \multicolumn{1}{c}{$D P$} \\
\hline Tempo de internação & 30 & 17,46 & 2,02 \\
Tempo total do uso do crack em anos & 30 & 6,97 & 5,92 \\
Tempo total do uso de maconha em anos & 28 & 14,00 & 9,22 \\
Tempo total do uso de cocaína em anos & 27 & 12,59 & 9,96 \\
Tempo total do uso do álcool em anos & 27 & 15,48 & 9,85 \\
Tempo total do uso de tabaco em anos & 24 & 15,21 & 10,64 \\
Tempo total de abstinência do crack em dias & 30 & 27,90 & 37,52 \\
Tempo total de abstinência da maconha em dias & 15 & 454,93 & 694,99 \\
Tempo total de abstinência da cocaína em dias & 14 & 428,36 & 479,22 \\
Tempo total de abstinência do álcool em dias & 22 & 183,41 & 507,79 \\
Tempo total de abstinência do tabaco em dias & 13 & 128,15 & 400,17 \\
\hline
\end{tabular}

\section{Resultados}

As variáveis sociodemográficas estão expressas na Tabela 1 , demonstrando que os grupos apresentaram características semelhantes.

A caracterização do grupo clínico, quanto ao padrão de uso de substância pode ser observada na Tabela 2 .

Na comparação quanto ao cálculo total, entre o GDC versus GNU, covariando para sexo, tempo de uso de crack e escore de intensidade de craving, o GDC obteve média de $-0,20(D P=$ $14,63)$; enquanto o GNU obteve média de 15,6; $(D P=22,24)$. Essa diferença foi significativa através da $\operatorname{ANCOVA}(F(4,45)=$ $2,77 ; p=0,040)$.

No teste para diferenças entre os blocos, evidenciou-se, de maneira geral, que os grupos apresentam diferenças ao longo das cinco blocos do IGT, sendo essas diferenças significativas no bloco $3(F(1,43)=12,56 ; p=0,005)$ e no bloco $5(F(1,43)$ $=4,38 ; p=0,042)$, por meio da ANOVA. Os escores médios e desvios-padrão por agrupamento de jogadas e por grupo podem ser visualizados na Figura 1.

Diante da Figura 1, é possível observar que o GDC manteve desempenho negativo e estável durante quase todo o jogo; apenas no bloco 3 para o bloco 4 houve um indício de sociodemográficas utilizou-se teste $t$ de Student e Qui-Quadrado. Para testar diferenças intra e entre grupos referentes ao cálculo total do IGT, foi conduzida uma análise de covariância (mixed-ANOVA). Na comparação intra e entre grupos quanto ao cálculo por blocos, realizou-se uma ANOVA com medidas repetidas. Para verificar diferenças entre os grupos referentes à classificação quanto ao desempenho na tarefa, utilizou-se o teste Qui-quadrado. (ANCOVA) acompanhando uma análise de variância mista

um processo aprendizagem, porém bem menos acentuada que o grupo controle.

A ocorrência de desempenho não-prejudicado, limítrofe ou prejudicado de cada participante durante a tarefa, baseado na classificação de Denburg et al. (2005), pode ser verificada na Tabela 3. Através do teste Qui-quadrado foram observadas diferenças significativas entre os grupos $(p=0,042)$ quanto à classificação do desempenho.

\section{Discussão}

De acordo com os resultados do presente estudo, o GDC apresentou desempenho significativamente inferior na tomada de decisão quando comparados ao GNU frente ao IGT. Os dados são reforçados quando se analisa a curva de aprendizagem de cada grupo. Enquanto o GNU apresentou uma aprendizagem durante o jogo, o GDC mostrou-se constante e negativo, havendo um indício de aprendizagem somente no final da tarefa. Esses achados estão de acordo com os demais estudos que avaliaram a tomada de decisão através do IGT em diferentes populações de usuários de substâncias (Cunha et al., 2011; Kim, Sohn, \& Jeong, 2011; Vadhan, Hart, Haney, van Gorp, \& Foltin, 2009; VerdejoGarcia et al., 2007). Além disso, estão de acordo com pesquisas 


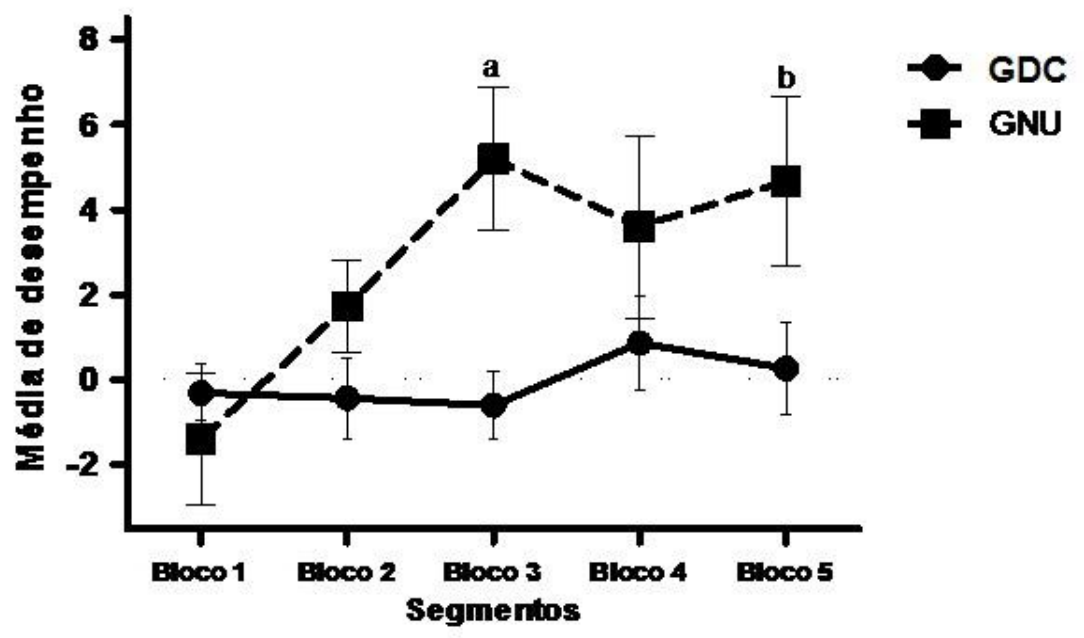

Figura 1. Média de desempenho dos grupos ao longo das jogadas do IGT. GDC - grupo dependentes de crack; GNU- grupo não usuário, $\mathrm{a}, \mathrm{b}=p<0,05$

Tabela 3

Distribuição de prejudicados, limitrofes ou não prejudicados por grupo

\begin{tabular}{lcc}
\hline & \multicolumn{2}{c}{ Grupos } \\
\cline { 2 - 3 } Classificação de Desempenho & GDC & GNU \\
\cline { 2 - 3 } & $N(\%)$ & $N(\%)$ \\
\hline Não prejudicados & $1(3,3 \%)$ & $7(46,6 \%)$ \\
Limítrofes & $24(80 \%)$ & $7(46,6 \%)$ \\
Prejudicados & $5(16,6 \%)$ & $1(6,6 \%)$ \\
\hline
\end{tabular}

prévias, em que o uso de substâncias se mostrou associado a um prejuízo nas funções executivas, (Ardila, Rosselli, \& Strumwasser, 1991; Eyler et al., 2009; Fernandez-Serrano, PerezGarcia, Perales, \& Verdejo-Garcia, 2010), mais especificamente nos componentes de controle inibitório (Verdejo-Garcia, LopezTorrecillas, Aguilar de Arcos, \& Perez-Garcia, 2005), memória de trabalho (Wareing, Fisk, \& Murphy, 2000) e fluência verbal (Cunha et al., 2004).

Os prejuízos na tomada de decisão dos participantes dependentes de crack podem refletir um viés de ausência de identificação, ou de prejuízos na percepção das consequências de suas escolhas, além da dificuldade destes sujeitos de aprenderem com seus próprios erros (Bechara, 2004). Tais prejuízos, associados à dificuldade dos usuários de cocaína e crack em lidarem com o craving, são fatores que podem influenciar no ciclo de recaídas típico da dependência. Neste sentido, o usuário que novamente sofre uma recaída, pode não perceber que isso influenciará, por exemplo, em uma futura potencial demissão do emprego, ou prejuízos financeiros e até desagregação familiar. Na possibilidade do usuário "perceber" as consequências de suas escolhas, também tem de controlar o impulso e o desejo de usar a substância frente aos sintomas de abstinência.

A tendência por viver apenas o momento, não levando em conta as consequências futuras, está associada com a diminuição do processamento top-down na tomada de decisão, na qual processos cognitivos superiores como as FE, exercem um "controle" diante das respostas de estruturas subcorticais como a amígdala, em uma espécie de "racionalização" do comportamento (Dalley, Everitt, \& Robbins, 2011). A hipótese de diminuição do "controle" pré-frontal pode, em parte, explicar o comportamento dos participantes do GDC durante a realização do IGT, quando o indivíduo abruptamente perde uma significativa quantia de dinheiro por jogar em um baralho ruim, mas não percebe o prejuízo, e segue optando pelas mesmas escolhas, manifestando certa "miopia para o futuro". Além disso, considera-se que os participantes do GDC, quando expostos ao crack pela primeira vez, poderiam já apresentar algum declínio nos processos de tomada de decisão e o consumo da substância se ajustaria como mais um elemento na equação que antecede e mantém a dependência. Em comparação a usuários de cocaína na forma inalada, tem-se mencionado que os usuários de crack estão associados a um padrão de consumo mais grave, com frequente envolvimento em atividades ilícitas, prostituição e alta probabilidade de viverem nas ruas, além de serem muito mais propensos a abandonarem o tratamento (Duailibi et al., 2008). Nesse sentido, os prejuízos evidenciados na tomada de decisão do GDC podem estar associados aos desfechos mencionados.

Outro fator importante, se refere às fases da adição em que os usuários se encontram, considerando que a dependência de substância se manifesta clinicamente como uma busca impulsiva pela droga, que ao longo do tempo avança para um comportamento compulsivo voltado ao consumo da substância. Nesse sentido, os indivíduos na fase de adição associada à impulsividade envolvem-se em opções desprovidas de planejamento, executadas, na maioria das vezes, de forma prematura sem julgamento apropriado em relação às consequências e riscos destas condutas (Patton, Stanford, \& Barratt, 1995). Isso pode ser expresso em uma ampla gama de 
comportamentos que podem envolver, sobretudo, a preferência por recompensas pequenas e imediatas (Evenden, 1999).

Com o uso crônico, os usuários aproximam-se de padrões compulsivos de consumo da substância que podem alterar o funcionamento típico de algumas regiões cerebrais, como à desregulação do sistema de recompensas. Este sistema é definido como a rede de circuitos que são ativados frente ao reforçamento positivo associado ao efeito da droga (prazer), através do aumento da liberação de dopamina. A hiperativação deste circuito impacta na diminuição do funcionamento do sistema de recompensas como um todo, possuindo como uma de suas consequências à exacerbação da síndrome de abstinência (Koob \& Le Moal, 2005). Desta forma, a fase da compulsão na dependência está associada a um poderoso componente motivacional na busca pela substância, em que estes comportamentos não são decorrentes apenas da procura incessante pelo prazer (recompensa), mas também da evitação do desprazer (sintomas de abstinência). A impulsividade e a compulsão pela droga podem alterar o funcionamento cognitivo, e esses efeitos podem contribuir para os prejuízos evidenciados no componente decisório.

Uma das limitações deste estudo refere-se ao fato de a maioria das participantes do grupo clínico ser usuário ou mesmo dependente de outras substâncias que também causam prejuízos cognitivos, como a maconha e o álcool. Este dado limita a afirmação de que os déficits no processo de tomada de decisão observados neste sentido tenham ocorrido exclusivamente em decorrência do uso de cocaína tipo crack. Além disso, o número de participantes, mesmo que igual ou maior do que os estudos encontrados com dependentes de cocaína e/ou crack, ainda é incipiente para a exploração dos múltiplos fatores que podem contribuir para os efeitos encontrados. Outro ponto importante é o fato de os dados referentes ao padrão de uso de substâncias, como o tempo de uso de crack, terem sido extraídos através de entrevista direta com os participantes, podendo não refletir uma total fidedignidade a respeito destas informações. Sugere-se que novas pesquisas sejam conduzidas com amostras maiores tanto no grupo clínico como no controle. Junto a isso, é importante que novas investigações utilizem um questionário de tomada de decisão para melhor entender o processo decisional dos participantes (Gorodetzky, Sahakian, Robbins, \& Ersche, 2011), além de correlacionar o IGT com outras medidas de desempenho de FE, bem como exame de respostas fisiológicas associadas à hipótese do marcador somático e o possível impacto destas variáveis na tomada de decisão.

\section{Referências}

American Psychological Association (APA). (2002). Diagnostic and statistical manual of mental disorders DSM-IV-TR (4를 $\mathrm{ed}$.). Washington: American Psychiatric Association.

Araujo, R. B., Pedroso, R. S., \& Castro, M. G. T. (2010). Adaptação transcultural para o idioma português do Cocaine Craving Questionnaire - Brief. Revista de Psiquiatria Clínica, 37(5), 195-198.

Ardila, A., Rosselli, M., \& Strumwasser, S. (1991). Neuropsychological deficits in chronic cocaine abusers. International Journal of Neuroscience, 57(1-2) 73-79.

Associação Brasileira de Empresas de Pesquisa - ABEP. (2010). Critério de classificação econômica - Brasil. Recuperado de www.abep.org/novo/Utils/
FileGenerate. $\operatorname{ash} x$ ?id $=46$.

Bechara, A. (2004). Disturbances of emotion regulation after focal brain lesions. International Review of Neurobiology, 62, 159-193.

Bechara, A. (2005). Decision making, impulse control and loss of willpower to resist drugs: a neurocognitive perspective. Nature Neuroscience, $8(11)$, 1458-1463.

Bechara, A., Damasio, A. R., Damasio, H., \& Anderson, S. W. (1994). Insensitivity to future consequences following damage to human prefrontal cortex. Cognition, 50(1-3), 7-15.

Bechara, A., \& Damasio, H. (2002). Decision-making and addiction (part I): impaired activation of somatic states in substance dependent individuals when pondering decisions with negative future consequences. Neuropsychologia, 40(10), 1675-1689.

Bechara, A., Damasio, H., Damasio, A. R., \& Lee, G. P. (1999). Different contributions of the human amygdala and ventromedial prefrontal cortex to decision-making. The Journal of Neuroscience, 19(13), 5473-5481.

Bechara, A., Damasio, H., Tranel, D., \& Damasio, A. R. (1997). Deciding advantageously before knowing the advantageous strategy. Science, 275(5304), 1293-1295.

Bechara, A., Damasio, H., Tranel, D., \& Damasio, A. R. (2005). The Iowa Gambling Task and the somatic marker hypothesis: some questions and answers. Trends in Cognitive Science, 9(4), 159-162.

Bechara, A., Tranel, D., \& Damasio, H. (2000). Characterization of the decisionmaking deficit of patients with ventromedial prefrontal cortex lesions. Brain, 123(11), 2189-2202.

Bechara, A., \& Van Der Linden, M. (2005). Decision-making and impulse control after frontal lobe injuries. Current Opinion in Neurology, 18(6), 734-739.

Berry, J., van Gorp, W. G., Herzberg, D. S., Hinkin, C., Boone, K., Steinman, L., \& Wilkins, J. N. (1993). Neuropsychological deficits in abstinent cocaine abusers: preliminary findings after two weeks of abstinence. Drug Alcohol Depend, 32(3), 231-237.

Cardoso, C., Núñez Carvalho, J. C., Cotrena, C., Schneider Bakos, D. G., Haag Kristensen, C., \& Paz Fonseca, R. (2010). Estudo de fidedignidade do instrumento neuropsicológico Iowa Gambling Task. Jornal Brasileiro de Psiquiatria, 59(4), 279-285.

Carvalho, J. (2010). Tomada de Decisão no Iowa Gambling Task: Estudos comparativos quanto às variáveis idade e escolaridade e estudo correlacional (Dissertação de Mestrado). Pontifícia Universidade Católica do Rio Grande do Sul, Porto Alegre.

Chan, R. C., Shum, D., Toulopoulou, T., \& Chen, E. Y. (2008). Assessment of executive functions: review of instruments and identification of critical issues. Archives of Clinical Neuropsychology, 23(2), 201-216.

Cordovil de Sousa Uva, M., Luminet, O., Cortesi, M., Constant, E., Derely, M., $\&$ de Timary, P. (2010). Distinct effects of protracted withdrawal on affect, craving, selective attention and executive functions among alcohol-dependent patients. Alcohol Alcohol, 45(3), 241-246.

Cunha, P. J., Nicastri, S., Gomes, L. P., Moino, R. M., \& Peluso, M. A. (2004). Neuropsychological impairments in crack cocaine-dependent inpatients: preliminary findings. Revista Brasileira de Psiquiatria, 26(2), 103-106.

Cunha, P. J., Bechara, A., de Andrade, A. G., \& Nicastri, S. (2011). Decisionmaking deficits linked to real-life social dysfunction in crack cocainedependent individuals. American Journal Addiction, 20(1), 78-86.

Dalley, J. W., Everitt, B. J., \& Robbins, T. W. (2011). Impulsivity, compulsivity, and top-down cognitive control. Neuron, 69(4), 680-694.

Damasio, A. R. (1996). The somatic marker hypothesis and the possible functions of the prefrontal cortex. Philosophical Transactions of The Royal Society B: Biological Sciences, 351(1346), 1413-1420.

Denburg, N., Tranel, D., \& Bechara, A. (2005). The ability to decide advantageously declines prematurely in some normal older persons. Neuropsychologia, 43(7), 1099-1106.

Duailibi, L. B., Ribeiro, M., \& Laranjeira, R. (2008). Profile of cocaine and crack users in Brazil. Cadernos de Saúde Pública, 24(4), 545-557. 
Evenden, J. (1999). Impulsivity: a discussion of clinical and experimental findings. Journal of Psychopharmacology, 13(2), 180-192.

Eyler, F. D., Warner, T. D., Behnke, M., Hou, W., Wobie, K., \& Garvan, C. W. (2009). Executive functioning at ages 5 and 7 years in children with prenatal cocaine exposure. Developmental Neuroscience, 31(1-2), 121-136.

Fernandez-Serrano, M. J., Perez-Garcia, M., Perales, J. C., \& Verdejo-Garcia, A. (2010). Prevalence of executive dysfunction in cocaine, heroin and alcohol users enrolled in therapeutic communities. European Journal of Pharmacology, 626(1), 104-112.

Folstein, M. F., Folstein, S. E., \& McHugh, P. R. (1975). Mini-mental state: a practical method for grading the cognitive state of patients for the clinician. Journal of Psychiatric Research, 12(3), 189-198.

Funahashi, S. (2001). Neuronal mechanisms of executive control by the prefrontal cortex. Neuroscience Research, 39(2), 147-165.

Gorodetzky, H., Sahakian, B. J., Robbins, T. W., \& Ersche, K. D. (2011). Differences in self-reported decision-making styles in stimulant-dependent and opiate-dependent individuals. Psychiatry Research, 186(2-3), 437-440.

Iraurgi Castillo, I., Trujols Albet, J., Jimenez-Lerma, J. M., \& Landabaso, M. (2009). Reliability and validity of the Spanish adaptation of the brief form of the Cocaine Craving Questionnaire-Now (CCQ-N-10). Adicciones, 2l(3), 195-202.

Kim, Y. T., Sohn, H., \& Jeong, J. (2011). Delayed transition from ambiguous to risky decision making in alcohol dependence during Iowa Gambling Task. Psychiatry Research, 190(2-3), 297-303.

Kjome, K. L., Lane, S. D., Schmitz, J. M., Green, C. M. L., Prasla, I., Swann, A. C., ... \& Moeller, F. G. (2010). Relationship between impulsivity and decision making in cocaine dependence. Psychiatry Research, 178(2), 299-304.

Koob, G. F., \& Le Moal, M. (2005). Plasticity of reward neurocircuitry and the 'dark side' of drug addiction. Nature Neuroscience, 8(11), 1442-1444.

Levine, B., Schweizer, T. A., O’Connor, C., Turner, G., Gillingham, S., Stuss, D. T., Manly, T., Robertson, I. H. (2011). Rehabilitation of executive functioning in patients with frontal lobe brain damage with goal management training. Frontiers in Human Neuroscience, 5, 9. doi: 10.3389/fnhum.2011.00009

Maia, A. L., Godinho, C., Ferreira, E. D., Almeida, V., Schuh, A., Kaye, J., \& Chaves, M. L. (2006). Aplicação da versão brasileira da escala de avaliação clínica da demência. Arquivos de Neuropsiquiatria, 64(2B), 485-489.

Nappo, S. A., Galduroz, J. C., Raymundo, M., \& Carlini, E. A. (2001). Changes in cocaine use as viewed by key informants: a qualitative study carried out in 1994 and 1999 in Sao Paulo, Brazil. Journal of Psychoactive Drugs, 33(3), 241-253.

Naqvi, N. H., \& Bechara, A. (2006). Skin conductance responses are elicited by the airway sensory effects of puffs from cigarettes. International Journal of
Psychophysiology, 61(1), 77-86.

Patton, J. H., Stanford, M. S., \& Barratt, E. S. (1995). Factor structure of the Barratt impulsiveness scale. Journal of Clinical Psychology, 51(6), 768-774.

Rosselli, M., Ardila, A., Lubomski, M., Murray, S., \& King, K. (2001). Personality profile and neuropsychological test performance in chronic cocaine-abusers. International Journal of Neurosciensce, 110(1-2), 55-72.

Schneider, D. D. G., \& Parente, M. A. M. P. (2006). O desempenho de adultos jovens e idosos na Iowa Gambling Task (IGT): um estudo sobre a tomada de decisão. Psicologia: Reflexão e Crítica, 19(3), 442-450.

Severtson, S. G., Hedden, S. L., Martins, S. S., \& Latimer, W. W. (2012). Patterns of cognitive impairments among heroin and cocaine users: the association with self-reported learning disabilities and infectious disease. Journal of Learning Disabilities, 45(2), 139-150.

Stout, J. C., Busemeyer, J. R., Lin, A., Grant, S. J., \& Bonson, K. R. (2004) Cognitive modeling analysis of decision-making processes in cocaine abusers. Psychonomic Bulletin and Review, 11(4), 742-747.

Tirapu-Ustárroz, J., García-Molina, A., Luna-Lario, P., Roig-Rovira, T., \& Pelegrín-Valero, C. (2008). Modelos de funciones y control ejecutivo (I). Revista de Neurología, 46(11), 684-692.

Toplak, M. E., Sorge, G. B., Benoit, A., West, R. F., \& Stanovich, K. E. (2010) Decision-making and cognitive abilities: a review of associations between Iowa Gambling Task performance, executive functions, and intelligence. Clinical Psychological Review, 30(5), 562-581.

Tversky, A., \& Kahneman, D. (1974). Judgment under uncertainty: Heuristics and biases. Science, 185(4157), 1124-1131.

Vadhan, N. P., Hart, C. L., Haney, M., van Gorp, W. G., \& Foltin, R. W. (2009) Decision-making in long-term cocaine users: effects of a cash monetary contingency on Gambling task performance. Drug and Alcohol Dependence, 102(1-3), 95-101.

Verdejo-Garcia, A. J., Lopez-Torrecillas, F., Aguilar de Arcos, F., \& Perez-Garcia, M. (2005). Differential effects of MDMA, cocaine, and cannabis use severity on distinctive components of the executive functions in polysubstance users: a multiple regression analysis. Addictive Behaviors, 30(1), 89-101.

Verdejo-Garcia, A., Benbrook, A., Funderburk, F., David, P., Cadet, J. L., \& Bolla, K. I. (2007). The differential relationship between cocaine use and marijuana use on decision-making performance over repeat testing with the Iowa Gambling Task. Drug and Alcohol Dependence, 90(1), 2-11.

Verdejo-Garcia, A., \& Bechara, A. (2009). A somatic marker theory of addiction. Neuropharmacology, 56 (1), 48-62.

Wareing, M., Fisk, J. E., \& Murphy, P. N. (2000). Working memory deficits in current and previous users of MDMA ('ecstasy'). British Journal of Psychology, 91(2), 181-188. 
Thiago Thiago Wendt Viola, graduando de Psicologia na Pontifícia Universidade Católica do Rio Grande do Sul, é bolsista de Iniciação Científica da Fundação de Amparo a Pesquisa do Estado do Rio Grande do Sul. Telefone: (51) 81366659. E-mail: thiago.viola@acad.pucrs.br

Caroline de Oliveira Cardoso, mestranda em Neuropsicologia Clínica e Experimental na Pontifícia Universidade Católica do Rio Grande do Sul, é psicóloga e bolsista do CNPq.

Ingrid D'Avila Francke, mestre em Psicologia pela Pontifícia Universidade Católica do Rio Grande do Sul, é psicóloga.

Hosana Alves Gonçalves, graduanda em Psicologia na Pontifícia Universidade Católica do Rio Grande do Sul, é bolsista de Iniciação Científica da Fundação de Amparo a Pesquisa do Estado do Rio Grande do Sul.

Julio Carlos Pezzi, doutorando no Programa de Pós-graduação Ciências da Saúde - UFCSPA, é psiquiatra.

Renata Brasil Araújo, doutora em Psicologia pela Pontifícia Universidade Católica do Rio Grande do Sul, é psicóloga e coordenadora do Programa de Dependência Química do Hospital Psiquiátrico São Pedro.

Rochele Paz Fonseca, doutora em Psicologia, é psicóloga, professora Adjunta na Pontifícia Universidade Católica do Rio Grande do Sul e coordenadora do Grupo de Neuropsicologia Clínica e Experimental.

Rodrigo Grassi-Oliveira, doutor em Psicologia, é psiquiatra, professor Adjunto na Pontifícia Universidade Católica do Rio Grande do Sul e coordenador do Grupo de Neurociência Cognitiva do Desenvolvimento. 